\title{
La labor antropológica en el campo de las políticas de extensión universitaria
}

\author{
Jimena Vázquez
}

Universidad de Buenos Aires

RESUMEN

Este artículo busca reflexionar sobre la labor antropológica en el campo de las políticas de educación superior, especificamente en el área de extensión universitaria, a partir de mi desempeño profesional en una universidad pública nacional de Argentina. Resumiré brevemente aspectos centrales de uno de los programas donde participo y el marco institucional del que formo parte. La selección del caso radica en su potencialidad para pensar la praxis antropológica, dado que condensa varias cuestiones que hacen a mi labor cotidiana y que atraviesan las diversas etapas o instancias en las que son producidas las politicas «extensionistas»: diseño/diagnóstico, planificación, ejecución y evaluación. Me interesa analizar la intervención del antropólogo o antropóloga en un área (la extensión universitaria) que emergió como el nexo entre la universidad y la comunidad, con el fin de contribuir a la transformación social desde una perspectiva que, sin embargo, hace (¿o hacía?) hincapié en la transferencia de conocimientos. Revisaré el concepto de extensión y las nuevas nociones que van ganando terreno. Como ejes para pensar la antropología aplicada, abordaré la distancia crítica y el involucramiento empático; la «aplicabilidad» de conceptos y metodologías etnográficas o la antropología como praxis, y finalmente, el trabajo interdisciplinario.

Palabras clave: antropología aplicada, praxis, extensión universitaria, políticas, trabajo interdisciplinario. 


\section{The Anthropological Work in the Field of University Extension Policies}

\section{ABSTRACT}

This article seeks to reflect on anthropological work in the field of higher education policy, specifically in the area of University Extension. This proposal tackles this issue from my own professional performance at a national public university in Argentina. I briefly summarize key aspects of one of the programs where I participated, as well as its corresponding institutional framework. The relevance of the case lies in its potential to reflect on anthropological praxis, and it encompasses several issues related to my daily work. Anthropological praxis crosscuts the various stages or levels which compose "extension» policies, namely design, diagnosis, planning, implementation and evaluation. My purpose is to analyze the anthropologist's intervention in this area -university extension-, that represents the "nexus» between the university and the "community», in order to contribute to social transformation. However, this perspective emphasizes (or probably emphasized?) knowledge transfer. I examine the notion of extension and the new concepts that are gaining ground. To think applied anthropology, I consider the following topics: the critical distance and the empathic involvement; the "applicability" of concepts and ethnographic methodologies, anthropology as a praxis; and, finally, interdisciplinary work.

Keywords: applied anthropology, praxis, universitary extension, policy, interdisciplinary work. 


\section{INTRODUCCIÓN: LA EXTENSIÓN UNIVERSITARIA}

La extensión universitaria a menudo es definida por las instituciones universitarias nacionales como la interacción entre academia y «comunidad» ${ }^{1}$, mediante la cual la universidad aporta a la sociedad — en forma crítica y creadora - los resultados y logros de su investigación y docencia, y por medio de la cual enriquece y redimensiona, a su vez, toda su actividad académica conjunta. De forma general, podemos pensar la extensión universitaria como el conjunto de las actividades conducentes a identificar los problemas y demandas de la sociedad, coordinar las correspondientes acciones de «transferencia» y reorientar las actividades de docencia e investigación a partir de la interacción con ese contexto.

El objetivo de la extensión universitaria es impulsar el acercamiento recíproco y la creación de vínculos de colaboración entre la universidad, las distintas instituciones gubernamentales y de la sociedad civil en general como parte integrante del colectivo social, generando un intercambio que permite no solo transmitir conocimiento sino ser permeable a recibir aportes que enriquezcan los procesos de enseñanza e investigación (Secretaría de Extensión Universitaria, Universidad Nacional de Avellaneda. El resaltado es mío).

Si bien hoy en día esta idea de extensión universitaria es ampliamente difundida y aceptada como base de toda política educativa en esta área, la consolidación de la noción ha atravesado un proceso de transformación permanente desde sus orígenes que está lejos de detenerse. En este apartado resumiré brevemente el surgimiento del concepto en Argentina y las nuevas nociones que están emergiendo cada vez con mayor fuerza, que problematizan no solo la idea de extensión sino las prácticas que conlleva, proponiendo nuevas formas de pensar la relación entre la universidad y la comunidad.

El surgimiento de la extensión universitaria se ha apoyado en diversas teorías pedagógicas de bases filosóficas diferentes que han apuntado a la idea de educación como proceso. Dentro de estas teorías se destaca la pedagogía cubana, que en el marco de la educación superior, tiene como objetivo la formación de

\footnotetext{
Entendida como categoría del sentido común y utilizada generalmente por dichas instituciones a modo de sinónimo del término sociedad (o bien como sinónimo de ideas como «sociedad civil», «medio social», etc.). Ver Universidad Nacional del Litoral - Proyectos de Extensión (www.unl.edu.ar); Universidad Nacional del Comahue, Secretaría de Extensión Universitaria (www.uncoma.edu.ar); Universidad Nacional de La Matanza, Secretaría de Extensión Universitaria (www.unlam.edu.ar), entre otras.
} 
profesionales capaces de transformar la sociedad a partir de su participación activa en la resolución de los problemas sociales (Pedroso, 2004).

En particular, en Argentina, este proceso tuvo su puntapié con la reforma universitaria de 1918, que constituyó un movimiento por la democratización de la enseñanza y cuyas reinvindicaciones reformistas bregaban, entre otras cuestiones, por la renovación de las estructuras y objetivos de las universidades, la implementación de nuevas metodologías de estudio y enseñanza, el compromiso con la realidad social: «con el principio de la extensión universitaria se pretendía extender la presencia de la universidad en la sociedad y relacionarla íntimamente con el pueblo» (Barbosa Illescas, 2008, p. 127). En otras palabras, al redimensionar los objetivos de la enseñanza superior e institucionalizar la extensión, la reforma comprometía a las universidades a desarrollar acciones que contribuyesen a modificar las condiciones de desigualdad.

En este nuevo contexto emergen, por un lado, la función social de la universidad pública nacional, entendida como puesta del saber universitario al servicio de la sociedad, haciendo de «sus» problemas un aspecto importante de su ocupación, y por el otro, la extensión universitaria como el pilar de la educación superior que tiene como fundamento y finalidad ese compromiso. A partir de la década de 1950, se desarrolla un período caracterizado por la orientación hacia una conceptualización de la extensión universitaria que garantizará la sistematicidad y coherencia de su función social, al concebirla como factor de cambio. La extensión universitaria pasa a ser entendida como promotora del desarrollo social de las «comunidades» con las cuales la universidad interactúa.

Estamos convencidos de seguir nuestro principio rector de apertura a la comunidad, con acciones que tienden a profundizar el rol social de la universidad en vistas de una sociedad plena y con justicia social (Secretaría de Extensión Universitaria, Universidad Nacional de La Matanza. El resaltado es mío).

A pesar de este reconocimiento sobre el rol político de la extensión, podemos puntualizar algunos aspectos que han caracterizado la extensión universitaria en Latinoamérica y que dificultan el logro de los objetivos de transformación social antes descritos: falta de presupuesto, marginación (frente a lo académico y la investigación), falta de preparación de los recursos humanos para su desarrollo, insuficiente estructura, falta de correspondencia entre planificación y acción extensionista, entre otros (Historia de la Extensión, VI Congreso Nacional de Extensión Universitaria, 2014). Me detengo brevemente en esta cuestión debido 
a que estos aspectos son permanentemente debatidos y puestos en evidencia en cada reunión, jornada y congreso de extensión en los que he participado, sin encontrar una sola institución en donde estas dificultades hayan sido sorteadas. Lo interesante, y que dejo para una futura reflexión, es por qué se enfatiza $-\mathrm{y}$ cada vez con más fuerza - el rol político de la universidad nacional —a través de la extensión-, pero permanecen situaciones (como los bajos presupuestos en comparación con otras áreas) que ponen un freno al desarrollo de ese rol.

Junto a las reflexiones en torno a los obstáculos mencionados, encontramos que la propia noción de extensión se encuentra hoy en debate debido a que en la práctica emerge una linealidad subyacente en donde hay un «experto» que tiene un conocimiento determinado y un «beneficiario» que recibe parte de ese conocimiento o ciertos beneficios de ese conocimiento. En lugar de promover el desarrollo de acciones extensionistas que surjan como respuestas a demandas explicitadas por diversos actores de la «sociedad $»^{2}$ como necesidades concretas, sucede que los proyectos y programas parecen surgir de un supuesto eje: la vulnerabilidad en la que se encuentran ciertos grupos excluidos y que los constituye como destinatarios privilegiados de las políticas extensionistas.

Como resultado de este cuestionamiento a la idea de extensión y las prácticas que conlleva, existen universidades nacionales que ya no cuentan con una Secretaría de Extensión Universitaria, sino con departamentos, direcciones, centros, áreas, que utilizan otros conceptos, como cooperación y servicios públicos (Universidad Nacional de Lanús), política y territorio (Universidad Nacional Arturo Jaureche), servicios a la comunidad (Universidad Nacional de General Sarmiento). Pero como podemos ver en estos escasos ejemplos, aunque no mencionen la idea de extensión permanecen ciertas nociones que darían cuenta de la relación (dialógica) entre la universidad y la comunidad que la extensión universitaria pretende representar. Dentro de estos campos de posibilidades, destaco la idea de responsabilidad social universitaria - RSU (Universidad Tecnológica Nacional), debido a que pone el foco en la responsabilidad ética que tiene la universidad para con la sociedad en la que se inserta, la necesidad de la construcción colectiva de los conocimientos y en la incorporación de los estudiantes universitarios como los actores principales de estas acciones (Vallaeys, 2008). Si bien la idea de responsabilidad social nació en el medio empresarial, quienes la utilizan proponen que su potencial uso da cuenta de «una nueva política de gestión»

2 El uso de comillas en este caso se debe a que utilizo la idea de sociedad como categoría nativa (ver nota al pie 1). 
que atiende «los impactos y efectos colaterales que se generan a diario dentro y afuera de la institución» (Vallaeys, 2008, p. 1). Este nuevo modelo está basado en fines éticos y de desarrollo social justo y sostenible, y por ello es aplicable a cualquier organización, incluso la universidad: «la responsabilidad social debe ser definida en términos de gestión de impactos y política de calidad ética en vez de compromiso social con los más necesitados, filantropía o solidaridad» (p. 2).

Este breve repaso sobre la idea de extensión y el proceso de transformación en el que se encuentra apunta a tres cuestiones. Primero, dar cuenta de los movimientos constantes que la atraviesan y que la distinguen del resto de los pilares universitarios precisamente por su «articulación» con la comunidad. Segundo, debido a la posibilidad de que esa noción condicione los objetivos y las posibilidades en cuanto al planeamiento y desarrollo de acciones extensionistas (no es lo mismo pensar la extensión como transferencia que como responsabilidad ética). Tercero, nos invita a que reflexionemos sobre los potenciales campos para el ejercicio profesional de la antropología, especialmente en los procesos de gestión pública vinculados con la producción de políticas educativas que tienen como fundamento central la transformación social. Subrayo esta idea de transformación social debido a su potencial relación con las ideas de desarrollo y bienestar, íntimamente relacionadas con los problemas y objetivos de la denominada antropología aplicada. Más adelante profundizaré en esta relación.

A continuación describiré algunos aspectos de mi experiencia profesional en una facultad orientada a las ciencias jurídicas en el conurbano bonaerense a través del análisis de uno de los programas en los que participo. Me interesa repasar el uso de algunas categorías usadas y legitimadas para pensar y llevar adelante proyectos y programas de «extensión universitaria» con relación al derecho. Finalmente, abordaré brevemente aspectos y potencialidades de la antropología aplicada.

\section{EXPERIENCIADEUNAANTROPÓLOGAENEXTENSIÓN UNIVERSITARIA}

A mediados del año 2011, comencé a trabajar en la Secretaría de Extensión Universitaria de una de las unidades académicas (vinculada con las ciencias jurídicas) de una universidad pública nacional de la República Argentina. Dicha institución forma parte del conjunto de universidades «nuevas» - algunas más y otras menos - cuyo proceso de emergencia se remonta a la década de $1970^{3}$,

En la década de 1970 se crean quince universidades nacionales, fundadas a partir de algunas existentes, subdivisiones, o bien nacionalizando instituciones privadas y públicas: Catamarca, 
fundado en la pretensión de descentralizar el sistema universitario del país (concentrado en la Universidad de Buenos Aires, la Universidad Nacional de La Plata y la Universidad Nacional de Córdoba). Este proceso continúa a finales de la década de $1980^{4}$ y se profundiza a partir del año $2000^{5}$, especialmente en el denominado «conurbano bonaerense», formado por veinticuatro partidos o municipios de la provincia de Buenos Aires que rodean el límite con la Ciudad Autónoma de Buenos Aires (también denominado Gran Buenos Aires). Esta idea de conurbano conlleva la visión de continuidad entre la Ciudad Autónoma de Buenos Aires y la Provincia.

Desde 1970 hasta la actualidad se han creado 43 universidades nacionales, once de las cuales se encuentran en el conurbano. El hecho de que las nuevas «camadas» de estudiantes sean en su mayoría primera generación de universitarios da cuenta de este proceso de apertura y democratización de la educación superior 6 , caracterizado por una proliferación de ofertas universitarias sobre carreras de pregrado, grado y posgrado, además de una amplísima variedad de actividades curriculares y extracurriculares (incluso «servicios») que se encuentran abiertas a la «comunidad».

Una de las particularidades de la universidad en la que me desempeño es su localización. Situada en el Gran Buenos Aires, comparte, con otras universidades del conurbano, aspectos tales como «fuerte articulación local, matrícula acotada, diversificación en la oferta de carreras, currículas abiertas y flexibles, políticas que tienden a la inclusión y permanencia de los estudiantes» (Zangrossi, 2013, p. 2). Lo interesante es que fueron «concebidas al servicio de los objetivos estratégicos de desarrollo de los distritos donde están insertas y sus zonas de

Santiago del Estero, Luján, Río Cuarto, Salta, San Luis, Lomas de Zamora, San Juan, Entre Ríos, Misiones, Comahue, Jujuy, Mar del Plata, Centro de la Provincia de Buenos Aires y La Pampa (ver Secretaría de Políticas Universitarias, Sistema Universitario).

4 Las universidades nacionales creadas entre 1988 y 1995 fueron diez: Quilmes, Villa María, La Matanza, General Sarmiento, Formosa, General San Martín, Lanús, Patagonia Austral, La Rioja y Tres de Febrero (ver Secretaría de Políticas Universitarias, Sistema Universitario ).

5 Entre 2000 y 2014 se crearon dieciséis universidades nacionales: Arturo Jaureche, Oeste, José C. Paz, Avellaneda, Chaco Austral, Alto Uruguay, Villa Mercedes, Tierra del Fuego, Río Negro, Rafaela, Comechingones, de las Artes, Hurlingham, Chilecito, Noroeste de la Provincia de Buenos Aires y Moreno (ver Secretaría de Políticas Universitarias, Sistema Universitario ).

6 Cabe hacer un apartado aquí para mencionar que, durante la dictadura cívico-militar que tomó el poder en marzo de 1976, se instauró un período de intervención de las universidades nacionales, así como de persecución, tortura y desaparición de estudiantes y docentes universitarios. Este hecho significó un descenso en la matriculación universitaria de alrededor del $22 \%$ (ver Zangrossi, 2013). 
influencia» (Hadida, 2015, p. 3). En esta nueva noción sobre el rol político que deben asumir las universidades nacionales, se subraya la idea de la educación como derecho colectivo y cuyo impacto debe estar dirigido a transformar las condiciones productivas y de desarrollo local y nacional (p. 3).

En este marco, comienzo a trabajar en extensión universitaria en tanto pilar de la universidad que pretende ser el «nexo» entre dos ámbitos: el universitario y el comunitario, a partir del desarrollo de acciones extensionistas que contribuyan a la transformación social. Recuerdo las primeras preguntas en el marco de la entrevista laboral que me hizo el secretario de ese entonces: ¿para qué sirve la antropología? ¿Cómo podrías contribuir con nuestros programas y proyectos? ¿Qué hace una antropóloga? Mi respuesta fue dar un gran resumen de los aportes que podría realizar desde la disciplina, refiriéndome a los métodos que podemos emplear para el abordaje de una problemática X (en retrospectiva, quizás debido a mi inexperiencia, en esa oportunidad hice hincapié en el empleo de una serie de técnicas cualitativas, como la entrevista y la observación, que me permitirían abordar un campo, un problema, de un modo que me diferenciase de otros profesionales). A su vez, el secretario me comentó cuáles eran los programas que tenían en curso, enfocados en su mayoría a la difusión de derechos. Estaban dirigidos a la comunidad — sin distinciones de ningún tipo (como edad, género, contexto local) - y eran ejecutados exclusivamente por docentes universitarios y graduados ${ }^{7}$. Pasada la entrevista, empecé a trabajar como contratada.

Una de las primeras actividades que me encomendó fue la revisión y lectura de uno de los programas que él consideraba central en la Secretaría: la promoción y difusión de derechos a través de referentes barriales. Este programa de promoción de derechos estaba en funcionamiento desde el año 2006. Antes de continuar, realizaré un breve resumen basándome en sus fundamentaciones originales (es decir, tal y como llegó a mis manos):

El problema general a resolver es restablecer los canales de información al ciudadano común, de modo tal que conozca y se apropie de las leyes y normas, y así de sus derechos y deberes en un Estado democrático. Logrando

7 Esto se debe a cómo se definen y piensan los programas (aunque hoy en día esta forma de conceptualización es eje de debate y reflexión, aún permanece). Más allá de las diferencias, en general estos programas tienen como actividad principal la realización de capacitaciones $\mathrm{y}$, como resultado, se da por sentado que quienes pueden llevarlas a cabo son los graduados y docentes que poseen título habilitante. Esta es una cuestión sumamente generalizada entre los sujetos con los que trabajo sobre cómo deben ser los programas y proyectos extensionistas, y más que nada sus actividades. 
cambios actitudinales en la vida comunitaria fomentando la participación y la acción para la resolución de conflictos y así mejorar su calidad de vida. Contribuyendo así a fortalecer un Estado de Derecho donde la población más vulnerable tenga acceso al poder que la información otorga. La comunidad sin distinción transita un problema crítico: la falta de información civil. Este problema general se traduce en conflictos particulares de diferente orden (fragmento de la fundamentación del programa. El resaltado es mío).

En otras palabras, su objetivo general es que los ciudadanos comunes logren acceder a la justicia (o mejoren ese acceso) a partir de su formación jurídica. Según el programa, los conflictos cotidianos $-\mathrm{y}$ específicamente aquellos que implican un estado de legalidad — pueden ser resueltos, o por lo menos, existe la posibilidad de actuar estratégica y activamente sobre su resolución. Sin embargo, el desconocimiento de las herramientas legales que permiten dicha resolución genera una desventaja que puede llevar a la dilatación —o bien a la no resolución — del problema: «los ciudadanos de una comunidad que desconocen de la norma, de las leyes, desconocen sus deberes y sus derechos y por ende ese desconocimiento los sumerge en una desventaja que muchas veces se suma a otras que jaquean seriamente su calidad de vida» (fragmento de la fundamentación del programa. El resaltado es mío). Ante este «problema», se busca capacitar al ciudadano común brindándoles aquellas herramientas conceptuales, procedimentales e informativas acerca de los derechos y obligaciones. Se pretende potenciar de esta forma la resolución de los problemas cotidianos, a través del trabajo solidario (voluntario) realizado dentro de su propio barrio (por medio de un consultorio) prestando ayuda en forma de asesoría jurídica: «darles a conocer a los ciudadanos la potencia del funcionamiento en red social. Fomentar el trabajo solidario como un beneficio para todos» (fragmento de la fundamentación del programa. El resaltado es mío). El objetivo de la instalación de los llamados «consultorios» en diversos barrios es facilitar el acceso efectivo a una fuente «confiable, conocida y cercana» ${ }^{8}$ :

Se tendrán en cuenta ciertos criterios básicos para la ubicación de los consultorios vecinales: comunidades con escasa capacidad de reacción ante situaciones de conflicto social; comunidades que se detecten con dificultades

\footnotetext{
Esto se debe a que hay barrios, como en el partido de La Matanza, localizados sobre la Ruta Provincial $\mathrm{n}^{\circ} 3$ (sobre todo a partir del km 35), que carecen de instituciones gubernamentales próximas y deben trasladarse hasta las localidades de San Justo, Cañuelas o La Plata, dependiendo del tipo de trámite de que se trate. Además que no hay muchas líneas de transporte público (colectivos) por lo que ese traslado implica, la mayoría de las veces, varias horas (entre traslado y atención).
} 
ambientales evidentes que afectan su calidad de vida; espacios de acceso fácil y económico para los habitantes del barrio (fragmento de la fundamentación del programa. El resaltado es mío).

Luego de una lectura exhaustiva, lo primero que me llamó la atención (como puede suponer el lector a partir de lo que he resaltado) fue la construcción de la alteridad. Como mencioné, en las definiciones de extensión universitaria suelen aparecer nociones como comunidad, medio social, sociedad y sociedad civil, que dan cuenta de una cierta homogeneidad ${ }^{9}$ cultural y social, pasando por alto la posibilidad de encontrar grupos heterogéneos. Lo novedoso fue que, en este caso, los destinatarios del programa eran definidos como ciudadanos comunes. Cabe aclarar que, en íntima relación con la idea de extensión (y con la producción de políticas en general), se habla de «destinatarios» de los programas y proyectos, enfatizando la posición receptiva en la que se encuentran ciertos grupos frente a la producción de aquellos. De manera inmediata asocié la idea de «ciudadanos comunes» con la formación académica de mis compañeros; sin embargo, a medida que fui interiorizando en el programa y el resto de las actividades que desarrollaba la Secretaría, empezaron a sedimentar algunas cuestiones que me resultaban centrales para entender los objetivos extensionistas articulados al derecho y a las formas de concebir la extensión: ¿quiénes eran «ciudadanos comunes»? ¿Quiénes eran «no comunes»? ¿Qué significaba «común» y hasta qué punto se era «común»? ¿Qué hacía que los «ciudadanos comunes» dejasen de serlo? ¿El objetivo era ese? ¿Por qué hablaban permanentemente en términos de «ciudadanía»? ¿De dónde surgía ese vocabulario? ¿Cómo se consolidaba y legitimaba? ¿Para pensar la «promoción de los derechos» era clave la categoría «ciudadanos comunes»? ¿Por qué? ¿La extensión implicaba provocar un pasaje de lo común a lo no común? ¿Cómo lograba esa provocación? ¿Cuál era, entonces, el objetivo de la extensión en este caso?

Esencialmente en lograr, en algún modo, democratizar este derecho, el derecho. Lograr que el mismo llegue a todos. Porque si bien hay principios jurídicos que dicen que la ley se presume conocida por todos, no hace falta ir muy lejos como para darse cuenta de que no es así y que la ignorancia de los derechos —o no sé si la ignorancia, sino el mal conocimiento de los derechos - se torna injusto de un lado y muchas veces abusivo por parte de

9 Salvo algunas excepciones que dan cuenta de la heterogeneidad de las formaciones sociales, especialmente aquellas universidades que utilizan conceptos alternativos a la idea de extensión. 
aquel que conoce el derecho (fragmento de entrevista a Mauro, coordinador del programa mencionado) ${ }^{10}$.

Durante los primeros meses de mi trabajo fue apareciendo una serie de conceptos que, aun estando ligados al derecho y a la ciudadanía, parecían adecuarse más a la idea de transferencia de conocimientos (y también de ciertos valores ${ }^{11}$ ) que subyace en la noción de extensión: democratizar, transmisión, socializar, fortalecer, acceso, mejorar, ignorancia o «mal conocimiento», desarrollo, concientizar... Estas ideas me conducían a visualizar dos actores jerarquizados, es decir, una relación lineal dada por la posesión desigual de saberes sobre el derecho y cómo acceder a la justicia: un actor universitario con conocimientos «expertos» y un actor - que llamaré «comunitario» (en correspondencia al uso que se hace del término «comunidad») — que no necesariamente carece de saberes, sino que ese saber puede ser un «mal conocimiento». Paulo Freire (1973) establece que la idea de extensión suele asociarse con: (i) un sujeto activo (el que extiende); (ii) la entrega (de conocimientos técnicos); (iii) el «mesianismo» (de quien extiende); (iv) la superioridad (del contenido de quien entrega) e inferioridad (de los que reciben), y (v) el mecanicismo (de la acción). Todas estas asociaciones de las que habla el autor, vinculadas con la idea de extensión como transferencia, las observaba en los informes de gestión, resúmenes y fundamentaciones de cada actividad y programa extensionista que llegaba a mis manos.

Fue en el cuestionamiento a esas definiciones, nociones y formas de producir la alteridad donde comencé a trabajar asiduamente. De hecho, en una de mis primeras reuniones, lo primero que pregunté a mis compañeros fue quiénes eran los «ciudadanos comunes» para ellos, o si había tipos de ciudadanía. Recuerdo que su primera reacción fue permanecer estupefactos por mi comentario, su intercambio de miradas, hasta que el secretario me respondió que «no sabía», que «nunca lo había pensado». A partir de ese momento empecé a trabajar sobre las nociones —muchas del sentido común, otras de la visión jerarquizada sobre diferentes

10 Esta entrevista, realizada en el año 2014, forma parte del trabajo de campo para mi investigación de Doctorado en Ciencias Antropológicas actualmente en curso en el Instituto de Altos Estudios Sociales - IDAES de la Universidad Nacional de San Martín. Para resguardar la identidad de los sujetos que forman parte del trabajo de campo usaré seudónimos, como en este caso.

11 Entre los valores más repetidos en informes de gestión, fundamentaciones de las actividades, materiales didácticos empleados como parte de folletería, etc., encontramos: «respeto», «solidaridad», «compromiso», «cooperación», «confianza», «contención», «ayuda», «responsabilidad». Incluso puede aparecer «amor». 
tipos de «saberes», etc.- que permanecían invisibilizadas, incuestionadas, en cada uno de los programas y acciones de la Secretaría. Esta naturalización, además, guiaba el planeamiento de las actividades propuestas. Estos planteamientos acerca de los sujetos «destinatarios» y cómo los concebíamos constituyeron (y constituyen) uno de los principales retos — si es válido llamarlos así- a los que me enfrentaba en las reuniones de equipo, en charlas con mis compañeros. Si bien quienes hacemos antropología trabajamos cotidianamente en relación con otras disciplinas, desde el uso de ciertos conceptos teóricos o modelos epistemológicos hasta la revisión crítica de esos conceptos, en el campo de la gestión el trabajo interdisciplinario es (¿o debería serlo?) permanente. La riqueza de esa interdisciplinariedad en la gestión radica, precisamente, en la posibilidad de articulación de propuestas teóricas y metodológicas para el abordaje de problemas de salud, vivienda, educación, transporte, patrimonio, entre otros.

Un aspecto interesante de esta construcción de la alteridad que no quiero dejar pasar es el uso implícito de una categoría que, sin embargo, es central para este y otros programas y proyectos de extensión: los vecinos. La relación vecino (categoría implícita)-ciudadano (categoría explícita) pareciera dar cuenta de la acción de transferencia producida por el programa en este caso. Los ciudadanos son individuos - considerados de modo abstracto y como jurídicamente igualesfrente a los vecinos - pensados como sujetos concretos, pero en un contexto de desigualdad jurídica-. Es en esta diferencia entre vecino y ciudadano donde se insertaría la extensión, que toma a ese vecino, a ese "par comunitario», lo capacita (se vuelve un referente jurídico) y lo ¿transforma? en un ciudadano «pleno», comprometido con «su comunidad».

Las discusiones de equipo no solo apuntaban a las formas de pensar la otredad, sino a los impactos potenciales o producidos por ese mismo programa (articulados, obviamente, a los objetivos, actividades propuestas y las concepciones sobre los sujetos a los cuales apuntan): ¿basta con solo «democratizar» el derecho, «extender» algunos conocimientos básicos, para que los destinatarios se vuelvan unos «ciudadanos plenos» y logren «acceder a la justicia»? Lo interesante del programa era no solo que formulaba un sujeto destinatario homogéneo definido por su supuesta vulnerabilidad sino que carecía de un diagnóstico que identificara las causas de esa «falta de conocimientos jurídicos» y permitiese evaluar los resultados posibles de las acciones propuestas. De hecho, los resultados ya se encontraban predefinidos en la idea de que, luego de conocer sus derechos, los ciudadanos comunes accederían a la justicia. 


\section{Cuadro 1. Esquema propio, resumen de los aspectos centrales del program a y la relación lineal entre ellos}

\begin{tabular}{|c|c|c|c|}
\hline $\begin{array}{c}\text { Destinatarios } \\
\text { predefinidos }\end{array}$ & $\begin{array}{c}\text { Actividades } \\
\text { propuestas }\end{array}$ & Resultados estimados & $\begin{array}{c}\text { Evaluación de } \\
\text { impactos }\end{array}$ \\
\hline \multicolumn{4}{|c|}{ 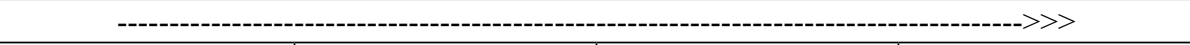 } \\
\hline $\begin{array}{c}\text { Ciudadano común } \rightarrow \\
\text { vecino }\end{array}$ & \multirow{4}{*}{$\begin{array}{l}\text { capacitación, difusión, } \\
\text { asesoría sobre consul- } \\
\text { tas recibidas, tutorías }\end{array}$} & Ciudadano & \multirow{4}{*}{$\begin{array}{l}\text { estadísticas generales } \\
\text { sobre consultas reci- } \\
\text { bidas por los sujetos } \\
\text { capacitados en forma } \\
\text { de fichas de consulta }\end{array}$} \\
\hline $\begin{array}{l}\text { Sujeto homogéneo } \rightarrow \\
\text { concreto }\end{array}$ & & $\begin{array}{l}\text { Sujeto homogéneo } \\
\text { abstracto }\end{array}$ & \\
\hline $\begin{array}{l}\text { Sin conocimientos } \\
\text { jurídicos } \rightarrow \\
\text { vulnerable }\end{array}$ & & $\begin{array}{c}\text { Con conocimientos } \\
\text { jurídicos } \rightarrow \text { acceso a } \\
\text { la justicia }\end{array}$ & \\
\hline Desigualdad jurídica & & Igualdad jurídica & \\
\hline
\end{tabular}

Cabe mencionar algunas cuestiones involucradas en el desarrollo del programa. Uno de los aspectos relevantes - y que hacen tanto a la producción de políticas como a la producción científica ${ }^{12}$ - es la aparición de ciertos valores considerados fundamentales para el ejercicio de la ciudadanía a partir del conocimiento de los derechos y el consecuente acceso a la justicia: «no quedarnos en la cuestión pura y exclusivamente jurídica, sino que el extensionista ${ }^{13}$ pueda desarrollar a su vez su costado social» (fragmento de entrevista a Mauro, coordinador del programa). Este fragmento nos acerca a la relación entre extensión (sobre todo la asociada a la transferencia) y valores: según Mauro, las prácticas involucradas en los proyectos y programas de extensión deberían promover incluso quizá facilitar- el desarrollo de un «costado social» de cada uno de los actores participantes, vinculado con el trabajo solidario, voluntario, comprometido, responsable. Es decir, no solo emerge una noción sobre qué debería ser la extensión, sino que aparecen ciertos valores relacionados con esa concepción. Pero no cualquier valor, sino ciertos valores como solidaridad, responsabilidad social, compromiso social, cooperación y participación, justicia para todos o justicia como valor democrático, que aparecen ligados a las ideas de extensión como la base institucional para la transformación y el desarrollo comunitario y un Estado de derecho. La relación que inmediatamente viene a la mente es lineal,

12 En especial, la idea que condensa esta articulación entre trabajo intelectual y valores es praxis.

13 La idea «extensionista» es una categoría nativa usada en el ámbito institucional donde trabajo para referirse a los sujetos que luego de ser formados jurídicamente se «transforman» en referentes jurídicos. Sin embargo, en otras instituciones universitarias se denomina así a los sujetos que trabajan en el área de extensión universitaria. 
donde encontramos unas necesidades normativas ${ }^{14}$ (Erwin, 2005) definidas por la universidad (la formación jurídica para poder acceder a la justicia y resolver problemas cotidianos) y unos valores vinculados con el derecho que se pretenden universalizables.

Me pregunto si el discurso institucionalizado sobre la extensión implica la definición de una vulnerabilidad a priori de un actor en particular -el comunitario-, que legitimaría no solo la propia noción de extensión sino las acciones desarrolladas en consecuencia. Al pensar en ese recorrido de la acción de extensión que va de lo universitario a lo comunitario y que transforma sobre todo al segundo en esa dirección, emergen dos cuestiones que buscan ser abordadas a través de la perspectiva RSU mencionada y que hoy se encuentra ganando terreno: la producción colectiva del conocimiento y el análisis del impacto producido por la implementación de las políticas universitarias de extensión.

Si bien había una evaluación de impactos a través del relevamiento estadístico sobre cuántas consultas recibía cada una de las personas previamente capacitadas, los motivos de esas consultas y los resultados alcanzados (es decir, si se había derivado la consulta al tutor por la profundidad y complejidad del problema consultado, si se había podido solucionar el problema sin la mediación del tutor, etc.), no existía un mecanismo que permitiese revisar y redireccionar las acciones propuestas. Además, las fichas de consulta (que completan quienes asesoran a otros) habían sido diseñadas por sociólogos sin consultar a los destinatarios sobre la potencial efectividad de esa ficha. Hoy no solo hemos modificado las fichas (en función de las demandas de los sujetos), sino que fuimos incorporando acciones tales como realización de entrevistas, elaboración de datos audiovisuales, mecanismos de contacto permanente a través de redes sociales, entre otras, que nos facilitan un contacto más fluido y con una mayor profundización sobre cuestiones que los actores consideran claves para su labor cotidiana y como promotores de derechos.

Retomo la pregunta sobre si la democratización del derecho (eje de la política de extensión de esta la institución donde me desempeño) conduce a una transformación del sujeto que recibe parte de ese conocimiento especializado y de la situación de desigualdad (de acceso a la justicia) en la que se encontraba previamente. Según el siguiente fragmento, se desprende que en el discurso

14 «Bradshaw identifies four types of needs - normative, felt, expressed and comparative. Normative needs are determined by the experts or administrators in charge of service. These are standards and have been established as desirable or ideal [...] they are presumed to de directly measurable» (Erwin, 2005, p. 77). 
institucionalizado esa transformación se da por el solo hecho de «extender los saberes» considerados por los expertos como aquellos necesarios para que la ciudadanía común pueda acceder a la justicia.

[El proyecto] que se ha dado como uno de sus objetivos generales: democratizar el derecho como una herramienta social promotora de mejor calidad de vida. Ha delineado como estrategia integrar y fortalecer referentes institucionales de la comunidad local en pos de llegar al ciudadano común, tomando como un instrumento mediador y facilitador de intervención en la comunidad al vecino como par comunitario otorgándole el plus de eficacia que otorga el conocimiento; desde esta conjunción acompañar a los pares a la resolución de conflictos diarios ha sido una labor constante y consecuente con el valor de la justicia para todos, la justicia como valor democrático (extracto del Informe de gestión 2009. El resaltado es mío).

Como propone Freire (1973), «en la medida en que, en el término extensión, está implícita la acción de llevar, de transferir, de entregar, de depositar algo en alguien, resalta en él una connotación indiscutiblemente mecanicista» (p. 26). Pero ese algo depositado, llevado, no es otra cosa que conocimiento y, en este sentido, «exige una presencia curiosa del sujeto frente al mundo. Requiere su acción transformadora sobre la realidad» (p. 28). Emerge nuevamente la pregunta por la transformación: ¿hay transformación? ¿De qué o de quiénes? ¿Cómo se produce? ¿Podemos pensar en una producción de políticas extensionistas donde se incluyan los saberes de los diferentes grupos de destinatarios, se elaboren mecanismos adecuados para el relevamiento de necesidades expresadas ${ }^{15}$ por los propios sujetos y acciones en consecuencia? ¿La extensión es (finalmente) el «puente» entre la academia y la sociedad (permitiendo su interacción y la producción colectiva de conocimientos con el fin último de lograr una transformación social)?

[Los referentes jurídicos] han ido más allá de los límites que puede tener el programa, que básicamente se trata de asesoramiento en cuestiones jurídicas básicas; han ido más allá, lo han juntado con cuestiones sociales, muchos han hecho ONG propias (fragmento de entrevista a Mauro, coordinador del programa mencionado).

Este breve relato sobre algunos aspectos del primer programa con el que trabajé profesionalmente, enfatizando el uso de ciertas categorías vinculadas con

15 De acuerdo con Erwin (2005), las necesidades expresadas (expressed needs) son aquellas que se vuelven demandas o peticiones de acción concretas por los grupos sociales (p. 77). 
la idea de extensión como transferencia y como eje universitario para la transformación social, buscó introducir al lector con los problemas epistemológicos que me encontré al trabajar como antropóloga en un área de gestión pública. No fueron (ni son) solo problemas epistemológicos (la relación entre teoría y aplicación, la relevancia de hacer investigación para poder generar proyectos de extensión que respondan a demandas explicitadas por los diversos actores con los que trabajamos) y conceptuales (el uso de ciertas categorías) con los que nos podemos encontrar, sino también metodológicos (la relevancia de incorporar técnicas cualitativas - etnográficas-, el tiempo disponible para hacer trabajo de campo) y éticos (¿cuáles son los límites para una distancia crítica? ¿Y para un involucramiento empático? ¿Es la extensión universitaria un paso clave para la transformación social y el desarrollo?).

A diferencia de esa experiencia concreta - un programa que venía en funcionamiento varios años antes de mi incorporación-, en los proyectos restantes participé y participo en su diseño, planeamiento y evaluación, e incluso en dos de ellos me desempeño como coordinadora. Esa participación diferenciada implica, a mi entender, una gran dificultad, porque no es lo mismo la labor que uno puede realizar al incorporarse a un programa o proyecto en curso (lo que conlleva que se puedan modificar algunos aspectos, pero no todo el programa, como las formas de evaluación, las actividades propuestas, los productos escritos) que la labor desde el minuto cero. Esto no debe llevar a suponer que la antropología es más adecuada —incluso más «profunda»— para el abordaje de diversos problemas o demandas sociales que otras disciplinas, sino que es necesario un trabajo articulado, generando espacios de discusión, reflexión e integrando las visiones de todos los actores involucrados para poder responder a esas demandas y proponer cambios en las condiciones estructurales de los grupos locales. Quizás aquí radique mi idea de compromiso empático para con los sujetos con los cuales trabajo, contribuyendo con aquellos aportes que, desde la antropología, puedo realizar.

En este sentido, considero que el campo disciplinar favorece un acercamiento más cercano a la propuesta de Freire para repensar la extensión, apuntando a cómo se producen los diversos saberes y cómo se ubica a los actores en esa producción (es decir, si se repite la linealidad antes planteada que va de lo universitario a lo comunitario o por el contrario, si se busca cuestionar esa relación unidireccional). 


\section{BREVE APARTADO SOBRE ACTIVIDADES Y METODOLOGÍA}

Antes de finalizar, creo necesario mencionar algunas cuestiones vinculadas con las actividades realizadas en el marco del programa mencionado y que se repiten en el resto de los programas y proyectos en los que participo. Las tareas son: relevamiento bibliográfico y de información diversa, reuniones de equipo a escala institucional y local, producción de informes escritos, producción de datos y documentos audiovisuales, entre otras. Algunas de las técnicas del trabajo de campo empleadas son: entrevistas semiestructuradas y en profundidad, revisión de fuentes secundarias, consulta de datos estadísticos oficiales, revisión de la normativa nacional y provincial, estadías en terreno, etc. Es importante destacar que la implementación de estas herramientas implicó e implica su ajuste a los objetivos de cada trabajo, a los aspectos sociales y territoriales, al financiamiento disponible y plazos de ejecución (en particular cuando hay articulaciones con otras instituciones).

Si bien las actividades no se acaban en aquellas mencionadas, sí son las que se repiten con mayor frecuencia y las que, de algún modo, se encuentran más vinculadas con la labor antropológica. A continuación, abordaré algunos aspectos a mi entender claves para pensar viabilidades y potencialidades para una antropología aplicada.

\section{¿Antropología aplicada o praxis antropológica?}

A lo largo de este artículo he intentado dar cuenta de mis primeras experiencias y sus consecuentes reflexiones en el campo de producción de políticas de educación superior vinculadas con el área de la extensión universitaria a partir de un caso empírico concreto. El objetivo ha sido dar cuenta de la situación con la que me encontré al comenzar a trabajar profesionalmente, debido a que condensa muchos aspectos de mi labor cotidiana: desde las reflexiones sobre la construcción de alteridad, las posibilidades de acción para la transformación, los marcos teóricos y epistemológicos de la extensión universitaria, el contexto donde se da esa acción extensionista y las potencialidades y posibilidades que ofrece el campo disciplinar, hasta las reflexiones éticas sobre los resultados esperados y los impactos producidos, entre otros.

Si bien hoy son muchos los antropólogos empleados por diversas organizaciones, permanece aún un cierto cuestionamiento sobre la idea de una antropología aplicada, una antropología en acción, una antropología del y para el desarrollo 
(denominaciones para una misma idea más allá de que plantean diferentes cuestiones y tienen orígenes distinto ${ }^{16}$ ) entendida como una «rama» de la disciplina caracterizada por la inserción profesional en campos que se encuentran por fuera de la academia. Es decir, una antropología orientada a producir cambios o transformaciones en el bienestar de diversos grupos sociales.

Antropología aplicada es la frase comúnmente utilizada por los antropólogos para describir sus actividades profesionales en programas que han tenido como principales éxitos cambios en el comportamiento humano, optando por contribuir a la mejora de los problemas sociales, económicos y tecnológicos, en vez de desarrollar una teoría social y cultural (Foster, 1969, p. 54).

En este sentido, la polémica que emerge entre una antropología «académica» y una antropología «aplicada» parece recaer sobre algunos ejes que considero relevantes mencionar aquí: (i) producción científica-valores; (ii) teoría-aplicación; (iii) tiempo político-tiempo etnográfico, y (iv) ética-práctica profesional. Debido a estos puntos de tensión que marcan el trabajo profesional, resulta interesante las posibilidades que abre la idea de praxis antes que una «antropología aplicada». ¿Por qué? Porque la aplicación de métodos y resultados de una investigación etnográfica no constituye un paso más, posterior a su concepción intelectual, sino que es parte de su epistemología (Mastrangelo, Díaz, Planes y Rodríguez, 2013). La antropología en tanto investigación científica es una práctica empapada de valores, de ética, de responsabilidad.

La aplicación de métodos y resultados forma parte de la actividad científica y no compartimientos estancos, diferenciados. Teoría y práctica se relacionan a través de la idea de praxis, como propone Gramsci: «El filósofo real no puede ser diferente del político, como hombre activo que modifica el ambiente entendiendo por tal el conjunto de relaciones en que cada uno de nosotros participa. Si la propia individualidad significa adquirir conciencia de estas relaciones.

16 La idea de una antropología aplicada comienza a vislumbrarse a principios del siglo XX de la mano de Malinowski, quien le atribuyó la función de consejería humanitaria durante la administración de África al gobierno británico. En Estados Unidos se crea en 1941 la Society for Applied Anthropology, cuyo principal aporte era la aplicación de métodos de campo para el desarrollo de intervenciones sociopolíticas orientadas a la resolución de problemas como fórmulas aplicables a diversas situaciones y contextos. En 1958, criticando esta noción, Sol Tax propone una «antropología de acción» sobre principios voluntaristas, rechazando la antropología aplicada como fuente de empleo. En paralelo, en la década de 1950 emerge la idea de una «antropología para el desarrollo» que, aunque fuertemente cuestionada, buscaba deconstruir los proyectos modernizadores definiendo su metodología desde una etnografía reflexiva. 
[Esto] implica modificar el conjunto de estas relaciones» (cit. por Kehoe, 2003, p. 5). Y es aquí donde surgen otras preguntas vinculadas con las posibilidades de lograr un distanciamiento crítico para optimizar el abordaje de una problemática, o bien cómo se articula (y si es posible) el distanciamiento y la empatía para con los diferentes actores y temas con los que uno trabaja.

Mi participación profesional en el área de extensión siempre es totalmente activa, y a veces es incorporada en un proyecto o programa en proceso (como el analizado), o bien desde sus inicios, además de participar en diversas actividades como «representante» de la institución (como en congresos, jornadas, reuniones con otros actores institucionales, etc.). Este posicionamiento conlleva a la reflexión cotidiana sobre las implicancias de esa participación, los límites institucionales que se encuentran o pueden encontrarse en el diálogo con otros actores, los sesgos que puede traer aparejados y las posibilidades de acción dentro del marco institucional. Lejos está negar las complejidades políticas de los contextos de intervención, ya que también hacen al contexto de producción de las políticas extensionistas (las instituciones universitarias) y del trabajo antropológico en general. En este sentido, creo que la idea de praxis permite abordar esa complejidad, asumiendo que la antropología es una práctica social.

Este trabajo buscó relatar mi experiencia profesional y los dilemas epistemológicos, teóricos, metodológicos y éticos que encuentro al hacer antropología aplicada. De allí la centralidad de mi voz en el relato que, no obstante, no es tal en el quehacer cotidiano, caracterizado por un intenso trabajo en equipo que incorpora - o pretende hacerlo - las voces de los otros (que será abordado en otra oportunidad). Considero que más que un problema es un desafío para pensar sobre la perspectiva de la praxis antropológica (aunque sigamos llamándola antropología aplicada) y las posibilidades de su desarrollo. Quizás la antropología aplicada pueda ser un equilibrio entre el distanciamiento crítico y el compromiso empático para aquellos profesionales que no solo producimos teoría social, sino cuya actividad cotidiana, enmarcada en la disciplina antropológica, genera diversos impactos que pueden o no contribuir a la transformación social. La pregunta que queda aún sin responder es si con nuestro accionar buscamos esa contribución y cuáles son, entonces, las consecuencias éticas de ese accionar. 


\section{REFERENCIAS BIBLIOGRÁFICAS}

Barbosa Illescas, Felipe (2008). Monografia histórica: la extensión universitaria en Iberoamérica y en Andalucía (España). Colección Observatorio Cultural $n^{\circ} 22$, Proyecto Atalaya. Madrid: Dirección General de Universidades de la Conserjería de Innovación, Ciencia y Empresa de la Junta de Andalucía.

Erwin, Alexander (2005). Applied Anthropology: tools and perspectives for contemporary practice. Boston: Pearson Education, Inc.

Freire, Paulo (1973). ¿Extensión o comunicación? La concientización en el medio rural. Buenos Aires: Siglo XXI.

Hadida, Sebastián (2015). La revolución silenciosa de las universidades del conurbano. Diario Bae, lunes 13 de abril, suplemento Negocios. Disponible en http://www.diariobae.com/notas/66868-la-revolucionsilenciosa-de-las-universidades-del-conurbano.html

Kehoe, Killian (2003). The Whole Picture - Gramscian Epistemology through the Praxis. Disponible en http://corkonlinelawreview.com/ editions/2003/2003ix.pdf

Mastrangelo, A., L. Días, C. Planes y M. Rodríguez (2013). Antropologías aplicadas varias: una revisión desde la praxis. Publicar, XI(XIV), junio.

Pedroso, Maricel (2004). Apuntes sobre el origen y evolución de la extensión universitaria en América Latina. Influencia de algunas teorías educativas contemporáneas en este proceso. Ponencia. IV Encuentro Nacional y I Latinoamericano: «La Universidad como objeto de investigación». Tucumán: Universidad Nacional de Tucumán.

Vallaeys, François (2008). La responsabilidad social universitaria: ¿cómo entenderla para quererla y practicarla? Brújula, 9(16), pp. 25-35. Lima: Asociación de Egresados y Graduados de la Pontificia Universidad Católica del Perú.

Zangrossi, Guillermo (2013). Las universidades del conurbano bonaerense. Impacto, desafíos y perspectivas. Ponencia. X Jornadas de Sociología. Facultad de Ciencias Sociales, Universidad de Buenos Aires. 


\section{Páginas web consultadas}

Secretaría de Políticas Universitarias, Sistema Universitario: http://portales. educacion.gov.ar/spu/sistema-universitario/ (última consulta 8/10/2015).

Universidad Nacional de Avellaneda, Secretaría de Extensión Universitaria: http://www.undav.edu.ar/ (última consulta 2/10/2015).

VI Congreso Nacional de Extensión Universitaria: http://extensionunr.edu.ar/ congresoextensionrosario2014-historiadelaextension.htm (última consulta 6/10/2015).

Universidad Nacional de La Matanza, Secretaría de Extensión Universitaria: http://www.unlam.edu.ar/index.php?seccion=4\&idArticulo=80 (última consulta 2/10/2015).

Universidad Nacional de Lanús: http://www.unla.edu.ar/index.php/secretariade-cooperacion-y-servicio-publico\#dir-de-cooperación (última consulta 2/10/2015).

Universidad Nacional Arturo Jaureche: https:/www.unaj.edu.ar/index.php/centro-de-politica-y-territorio (última consulta 2/10/2015).

Universidad Nacional de General Sarmiento: http://www.ungs.edu.ar/ms_centro_servicios/ (última consulta 2/10/2015).

Universidad Tecnológica Nacional: http://www.seu.utn.edu.ar/extension/index. php (última consulta 2/10/2015).

Pontificia Universidad Católica del Perú, Políticas de Responsabilidad Social Universitaria: http://dars.pucp.edu.pe/publicacion/politicas-de-responsabilidad-social-universitaria/ 